\title{
Six-day westward propagating wave in the maximum electron density of the ionosphere
}

\author{
D. Altadill ${ }^{1}$, E. M. Apostolov ${ }^{1}$, Ch. Jacobi ${ }^{2}$, and N. J. Mitchell ${ }^{3}$ \\ ${ }^{1}$ Observatorio del Ebro, (URL - CSIC), Horta Alta 38, E43520 Roquetes, Spain \\ ${ }^{2}$ Institute for Meteorology, University of Leipzig, Stephanstr. 3, 04103 Leipzig, Germany \\ ${ }^{3}$ Department of Electronic and Electrical Engineering, University of Bath, BA2 7AY, Bath, UK
}

Received: 23 August 2002 - Revised: 20 December 2002 - Accepted: 11 February 2003

\begin{abstract}
Analyses of time-spatial variations of critical plasma frequency $f o \mathrm{~F} 2$ during the summer of 1998 reveal the existence of an oscillation activity with attributes of a 6-day westward propagating wave. This event manifests itself as a global scale wave in the $f o \mathrm{~F} 2$ of the Northern Hemisphere, having a zonal wave number 2 . This event coincides with a 6-day oscillation activity in the meridional neutral winds of the mesosphere/lower thermosphere (MLT). The oscillation in neutral winds seems to be linked to the 6-7-day global scale unstable mode westward propagating wave number 1 in the MLT. The forcing mechanisms of the 6-day wave event in the ionosphere from the wave activity in the MLT are discussed.
\end{abstract}

Key words. Ionosphere (Ionosphere-Atmosphere interactions; Mid-latitude Ionosphere) - Meterology and atmospheric dynamics (waves and tides)

\section{Introduction}

Planetary waves (PW) are a common feature in the wind and temperature regimes of the Mesosphere/Lower Thermosphere (MLT). Their periods coincide with the theoretically predicted Rossby normal modes of the atmosphere (Salby, 1984): 2-, 5-, 10-, and 16-day waves. From these waves, the 2- and 5-day waves are mainly a summer phenomena in the MLT winds and they are predominantly westward propagating waves (Harris, 1994; Wu et al., 1994). Recent investigations also show the existence of long period oscillations in the MLT with periods out of the expected range for PW, especially the so-called "6-7-day wave". Kamalabadi et al. (1997) and Riggin et al. (1997) report westward propagating 6.5-day waves with zonal wave number $s=1$ in the MLT at equatorial and middle latitudes. Meyer and Forbes (1997a) concluded that the westward 6.5-day wave is a global scale unstable mode and Clark et al. (2002) came to a similar con-

Correspondence to: D. Altadill

(daltadill@obsebre.es) clusion for the 7-day wave westward propagating wave number 1 . The wave-wave interaction or the modulation of the upward propagating tides and gravity waves by the PW activity in the MLT is, among other plausible mechanisms, a likely cause of the transmission of PW activity to the ionosphere (Forbes, 1996; Pancheva et al., 2002). Although it is accepted that the major cause of the variability of the ionosphere is due to solar activity (UV and EUV) and to geomagnetic activity, the contribution to the ionospheric variability transmitted from lower atmospheric levels can generate a comparable contribution to that of geomagnetic origin (Risbeth and Mendillo, 2001). Forbes et al. (2000) quantified the degree of variability of the ionospheric F-region due to periods of PW (2-30 day) to be about $15-20 \%$ under quiet geomagnetic conditions. Apostolov et al. (1998) showed that the contribution to the variability of the ionospheric F-region due to PW type oscillations is larger in summer solstice.

PW type oscillations with a period of about 6 days exist in the electron density of the lower and upper ionosphere (Pancheva and Lastovicka, 1998; Altadill, 2000; and references therein). The oscillations with a period of about 6 days in the F-region have similar characteristics to that reported for the "6-7-day wave" in the MLT; their period ranges from 135 to $170 \mathrm{~h}$, and their oscillation activity is largest in the summer half year (Apostolov and Altadill, 1996; Apostolov et al., 1998). However, the oscillation activity with a period of about 6 days has not been studied as extensively as the quasi-2-day oscillations in the ionosphere. Significant similarities have been found between the quasi-2-day oscillations in the ionospheric F-region and the 2-day wave in the MLT, especially at middle latitudes. They have the same oscillating period range, their oscillation activity is largest in the summer half year, and they have a westward direction of propagation (zonal wave number $s=3$ in the MLT and $s=1$ in the ionosphere), and their vertical wavelengths are larger than $100 \mathrm{~km}$ (Harris, 1994; Apostolov et al., 1995; Wu et al., 1996; Forbes et al., 1997; Altadill and Apostolov, 1998). Moreover, a wide number of studies show the possible indirect influence of the 2-day wave in the MLT on 
the quasi-2-day oscillations in the ionosphere (Forbes and Zhang, 1997; Altadill et al., 1997; Pancheva et al., 1994). The large enhancements of the geomagnetic activity related to storms could also generate wave-like oscillations with a period of 2 days in the ionosphere (Altadill and Apostolov, 2001; Pancheva et al., 2002).

Recent results indicate the presence of vertical propagating 6-day wave events in the middle latitude F-region (Altadill and Apostolov, 2001; Altadill et al., 2001b). They showed there that some of these events propagate from below into the ionosphere, likely being forced by 6-day wave events in the MLT winds. Others display downward energy propagation and they coincided with geomagnetic activity variations related to storms. However, the above results were based on the analysis of a single ionospheric station. Preliminary results about the zonal structure of the 6-day wave events in the ionosphere (Altadill et al., 2001a) indicate that these wave structures manifest themselves frequently in the ionosphere with characteristics of stationary or westward traveling waves, mainly during the summer half year. However, the existence of 6-7-day waves at global or large scale forced by PW activity in the MLT has not been proved in the ionosphere.

The aim of this case study is to show the global scale pattern of a 6-day oscillation event in the F-region ionosphere that manifests itself during the summer of 1998 and to make an attempt to determine its origin from the wave activity in the MLT.

\section{Data and analysis}

Our study is performed for the time interval 20 May - 17 September 1998, when the ionospheric F-region displayed large oscillation activity at periods of about 6 days (Altadill et al., 2001b). To obtain information of the dynamics in the MLT region, we used hourly records of neutral wind in the UK $\left(51.7^{\circ} \mathrm{N}, 1.8^{\circ} \mathrm{E}\right)$ made by meteor radar and LF D1 wind measurement performed at Collm $\left(52^{\circ} \mathrm{N}, 15^{\circ} \mathrm{E}\right)$. The UK radar has been described by Muller et al. (1995) and details of the instrument and technique for LF D1 wind measurements have been described by Schminder and Kürschner (1994). The wind data above UK is mainly recorded at an altitude of about 92-95 km and the LF D1 wind changes its altitude of measurements between $82 \mathrm{~km}$ during daylight hours to $95-105 \mathrm{~km}$ at night, due to the daily variations of the lower ionosphere. Then, for analysis we discarded the Collm data recorded below $85 \mathrm{~km}$ in order to have a similar altitude of measurement at the two wind stations.

To study the ionospheric variability, we use hourly values of the plasma frequency at $240 \mathrm{~km}$ and $h m \mathrm{~F} 2$ obtained at the Observatorio del Ebro station $\left(40.8^{\circ} \mathrm{N}, 0.5^{\circ} \mathrm{E}\right)$ by the ionogram inversion algorithm described by Huang and Reinisch (1996). To find these parameters, the ionogram's traces were carefully revised in order to avoid spurious data of the automatic scaling. To evaluate the spatial pattern of the oscillation activity in the ionosphere, we used the $f_{o} \mathrm{~F} 2$ of 23
Table 1. List of the ionospheric stations used in the analysis

\begin{tabular}{llll}
\hline URSI code & $\begin{array}{l}\text { Geographical } \\
\text { Latitude }\end{array}$ & $\begin{array}{l}\text { Geographical } \\
\text { Longitude }\end{array}$ & $\begin{array}{l}\text { Geomagnetic } \\
\text { Latitude }\end{array}$ \\
\hline EB040 & 40.8 & 0.5 & 43.6 \\
RO041 & 41.8 & 12.5 & 42.3 \\
JR055 & 54.6 & 13.4 & 54.3 \\
UP158 & 59.8 & 17.6 & 58.3 \\
VT139 & 40.7 & 17.9 & 39. \\
SQ143 & 42.7 & 23.4 & 41.0 \\
LD160 & 60.0 & 30.7 & 56.1 \\
MO155 & 55.5 & 37.3 & 50.4 \\
AS237 & 37.9 & 58.3 & 30.4 \\
NS355 & 54.6 & 83.2 & 44.2 \\
BP440 & 40.0 & 116.3 & 28.8 \\
ML449 & 49.6 & 117.5 & 38.4 \\
SN437 & 37.2 & 127.1 & 30. \\
MG560 & 60.0 & 151.0 & 50.9 \\
PK553 & 53.0 & 158.7 & 44.9 \\
PA836 & 34.6 & 239.4 & 42.3 \\
EG931 & 30.4 & 273.3 & 41.1 \\
WP937 & 37.9 & 284.5 & 49.2 \\
PRJ18 & 18.5 & 292.8 & 29.8 \\
SMJ67 & 67.0 & 310.0 & 77.1 \\
NQJ61 & 61.2 & 314.6 & 70.9 \\
EA036 & 37.1 & 353.3 & 41.4 \\
RL052 & 51.6 & 358.7 & 54.1 \\
\hline
\end{tabular}

ionospheric stations longitudinally distributed in the Northern Hemisphere (Table 1). The stations were selected so as to have the largest longitudinal distribution and good quality data, thereby assuring as much as possible a continuous time series with minimum gaps. With this longitudinal distribution we are able to obtain the zonal structure of the oscillation activity in the $f o \mathrm{~F} 2$, if any. We also used the 3-hourly $A_{p}$ index of geomagnetic activity and the hourly values of the geomagnetic index $D_{s t}$, in order to take into account the geomagnetic conditions during the events of oscillation. This data was obtained from the Solar-Terrestrial Physics Division of the National Geophysical Data Center (http://www.ngdc.noaa.gov/stp/).

In order to determine the existence of oscillations in the above geophysical parameters, we use statistical methods based on spectral analysis, particularly the Lomb-Scargle periodogram method (Scargle, 1982; Horne and Baliunas, 1986) and the Rayleigh power analysis (Bai and Cliver, 1990). These methods are useful tools for evaluating periodic components in time series with data gaps. Moreover, the relationship between the Rayleigh power and the maximum likelihood methods (Bai, 1992) and the cumulative property of the logarithm of the probability function enables us to determine intervals when a periodic component is really in operation. We also used bispectral analysis technique (Kim and Powers, 1979), in order to investigate the effects of the nonlinear wave-wave coupling in the ionospheric and MLT parameters. 


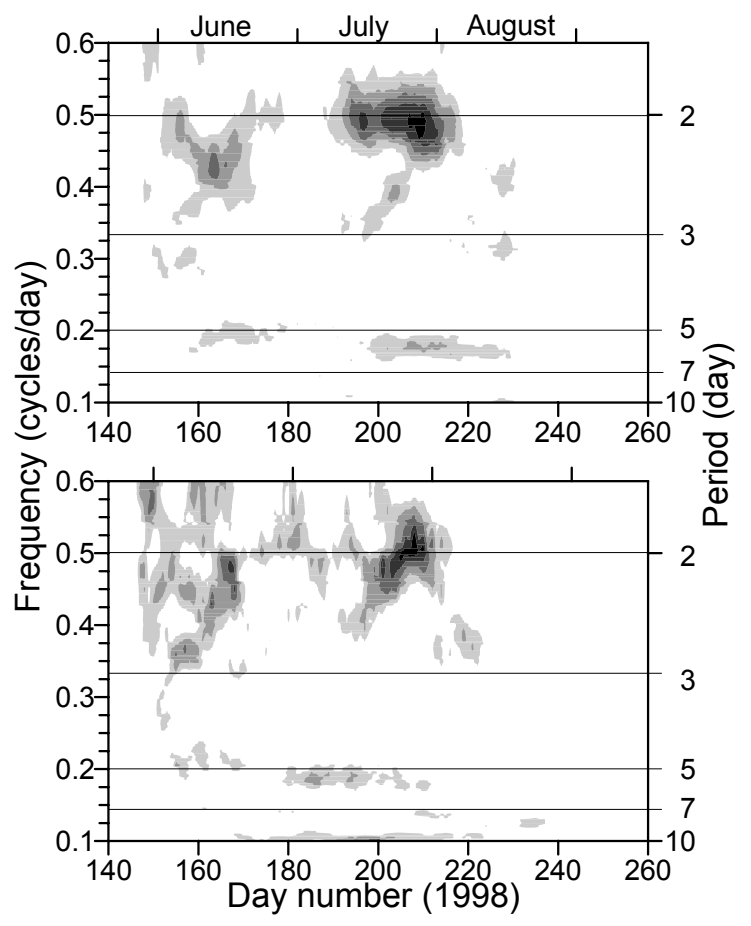

Fig. 1. Frequency-time cross sections of the power spectra of the meridional wind component in the MLT for UK (top) and Collm (bottom) wind stations.

\section{Six-day oscillation in the MLT neutral winds}

Figure 1 shows the dynamic spectra of meridional wind recorded at UK (top) and Collm (bottom) in the periodic range from 1.7 to 10 days for the time interval from 20 May to 17 September 1998. These contour plots depict only the statistically significant signal of the normalized power spectra computed by the Lomb-Scargle periodogram, specifically those frequencies that have a power above the 0.95 confidence level. Looking at the plots, two strong events of 2-day oscillation can be distinguished in the meridional wind, during June and July, that is better expressed in the data from UK. Also, a 6-day oscillation activity is observed in both data sets, from middle July to middle August as seen from UK data, and during July from the Collm data. Although not shown here, we obtained the dynamic spectra for the zonal wind component as is done for the meridional. We found the same strong events of 2-day oscillation in Collm data, whereas UK data displayed 2-day oscillation activity only in July. We did not find significant oscillation activity in zonal winds at periods of about 6 days in the UK or in the Collm data.

To assess the time interval when the 6-day oscillation is really in operation within the time interval from day 140 to 260, we apply the relationship between the Rayleigh power and the maximum likelihood methods (Bai, 1992). First, we compare the power spectrum obtained by Lomb-Scargle and Rayleigh methods for the meridional wind data from UK (Fig. 2). The results obtained by these methods are equiv-

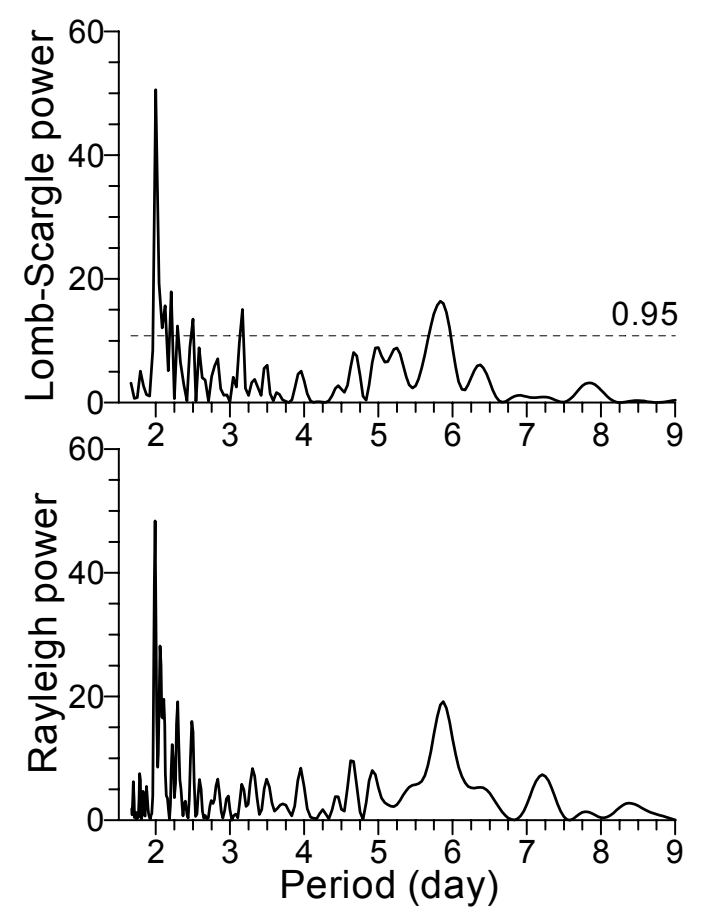

Fig. 2. Normalized power spectrum obtained by Lomb-Scargle method (top) and Rayleigh power (bottom) obtained for the UK meridional wind data in the time interval from 17 May to 17 September 1998.

alent and they show a large peak at the 2-day period and a second important peak at the 6-day period, both peaks above the 0.95 confidence level. Jacobi et al. (2001) have already found oscillation activity at periods of about 6 days in the MLT winds during July-August 1998, so we confirm this using different methodology. The oscillation activity we are interested in is the 6-day oscillation and the dominant period obtained by the Rayleigh method is 5.9 days. We may obtain then the time development of the logarithmic likelihood function for the aforementioned oscillation. The logarithmic likelihood is a cumulative function,

$m\left(t_{k}\right)=\sum_{i=1}^{k} \ln \left[1+A \cos \left(2 \pi v_{0} t_{i}-\theta_{0}\right)\right]$,

where $v_{0}$ is the frequency under study, $A$ is its Rayleigh amplitude, $\theta_{0}$ its phase, and the index $k$ indicates the number of measurement. Bai (1992) provide clear explanations of the meaning of the magnitudes expressed in Eq. (1). Therefore, the logarithmic likelihood oscillates or decreases when the oscillation is not active and it increases in the time intervals when the oscillation is in operation.

The results are plotted in Fig. 3, where the smoothed wind data with a 51-h running mean has been added to compare better the time interval of existence. We observe in Fig. 3 a sharp increase in the logarithmic likelihood from day number 192 to 222 that coincides with an enhancement of oscillation activity with a period of 5.9 days, as seen from the 


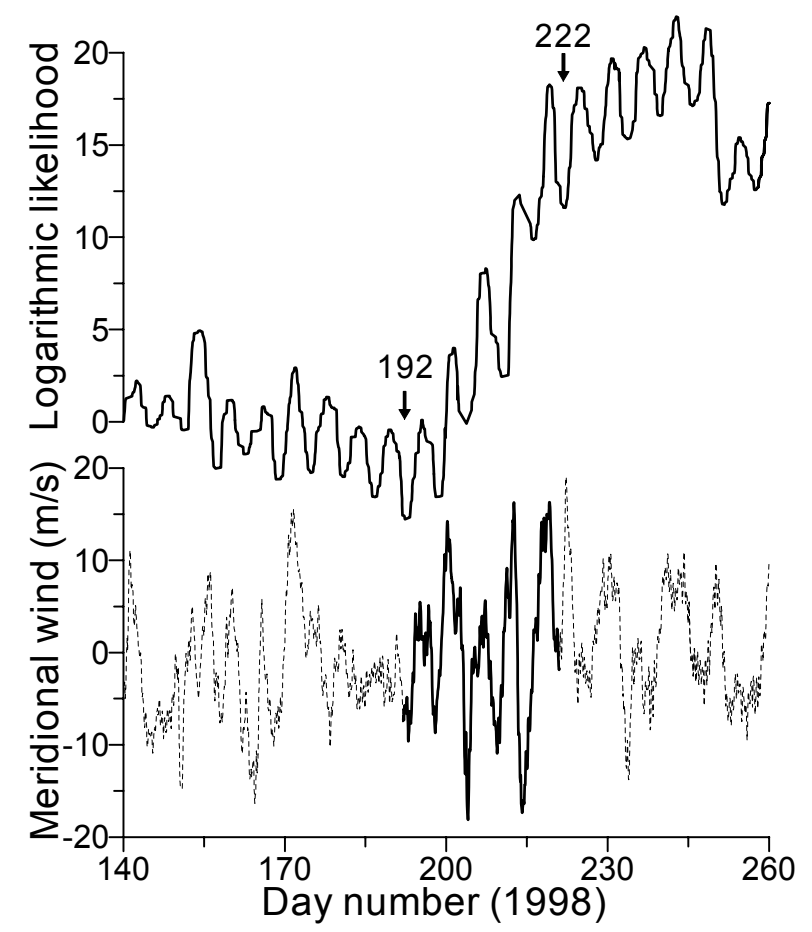

Fig. 3. Time development of the logarithmic likelihood for the period of 5.9 days in the UK meridional wind (top) and of the 51-h running mean values of the UK meridional wind (bottom). The results are plotted for the time interval from 17 May to 17 September 1998. The arrows in the top plot indicate the time interval when the oscillation is in operation. The thick line in the bottom plot enhances this interval.

smoothed data. Within this time interval we clearly observe 5 waves. So, we proved the existence of a 6-day oscillation in the MLT winds above UK from 11 July to 10 August 1998. Later, from day number 222 to 246, the logarithmic likelihood also increases, but very slowly, so that its existence in the last time interval is doubtful. This is confirmed by observation in the smoothed data, because after day 222 it remains an oscillation activity, but the wave crests are detached more than 6 days to each other. We obtain similar results from MLT winds above Collm (Figs. 4-6). We tried to evaluate the zonal propagation of this oscillation as the result of a westward propagating wave with zonal wave number 4 . However, this result may be spurious because we have only two wind stations that are very close to each other and because of the different type of measurements. Whereas the wind data above UK is recorded at an altitude of about $92-95 \mathrm{~km}$, the LF D1 wind changes its altitude of measurements between $85 \mathrm{~km}$ up to $105 \mathrm{~km}$. Then a small error in the phase difference between the two stations could provide a large error in the wave number estimate. Because of that, we cannot perform a precise evaluation of the wave structure of this 6-day event in the MLT winds. With the data we use, we may only speculate about it. However, wave structures with a period of 6-7 days have been found in the wind regime of the MLT (Kamalabadi et al., 1997; Riggin et al., 1997) with a west-

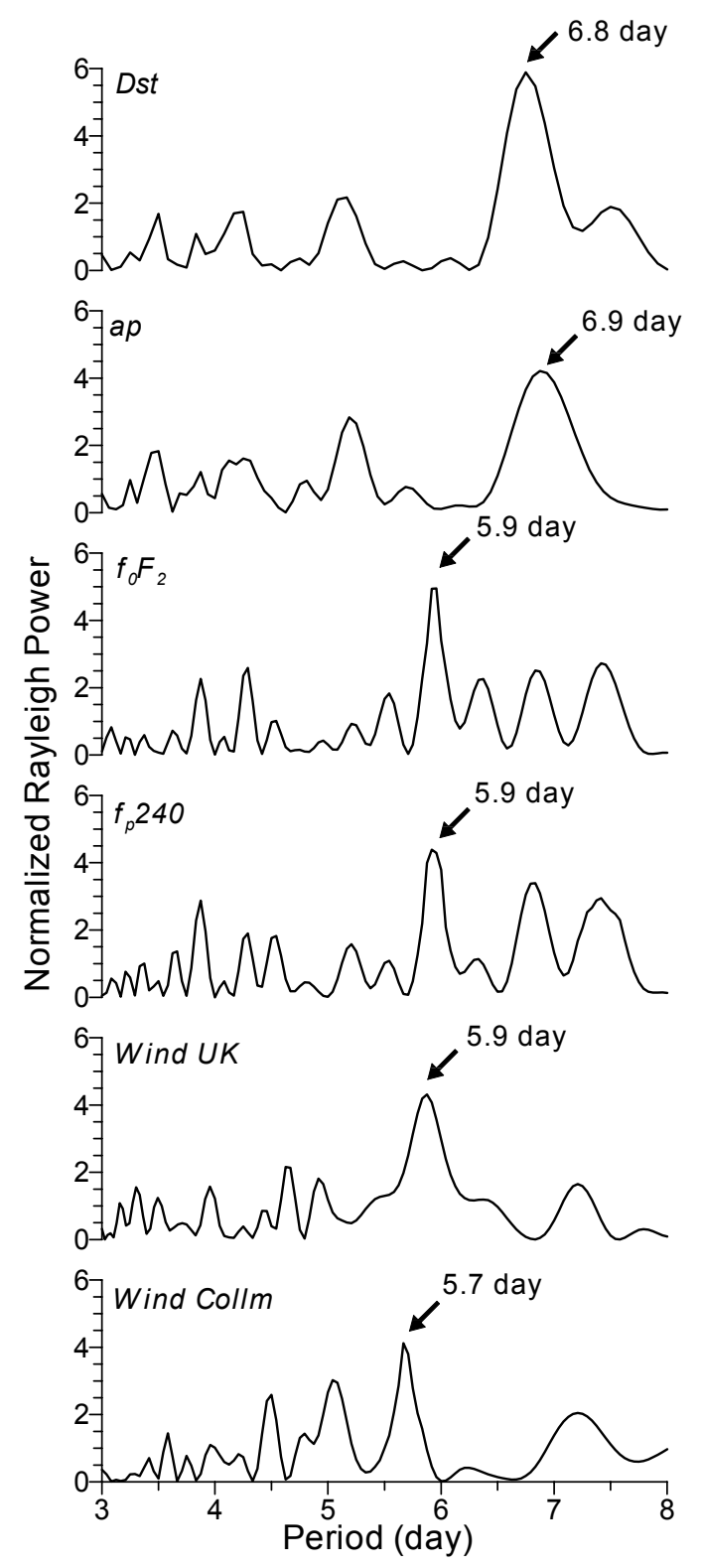

Fig. 4. Normalized Rayleigh power computed for the indicated time series in the time interval from 1 June to 31 August 1998. Arrows indicate the dominant period of oscillation of each time series.

ward propagating wave number 1 and it has been concluded that these waves are global scale unstable modes (Meyer and Forbes 1997a; Clark et al., 2002). So, the oscillation activity of a period of about 6 days that we observed could probably be the effect of the aforementioned wave structure.

\section{Simultaneous 6-day oscillation in the MLT and iono- spheric parameters}

Figure 4 depicts the power spectra obtained by the Rayleigh method of indicated time series for the time interval from 1 June to 31 August 1998. The ionospheric data used in this analysis is from Observatorio del Ebro, and $f_{p} 240$ refers to 

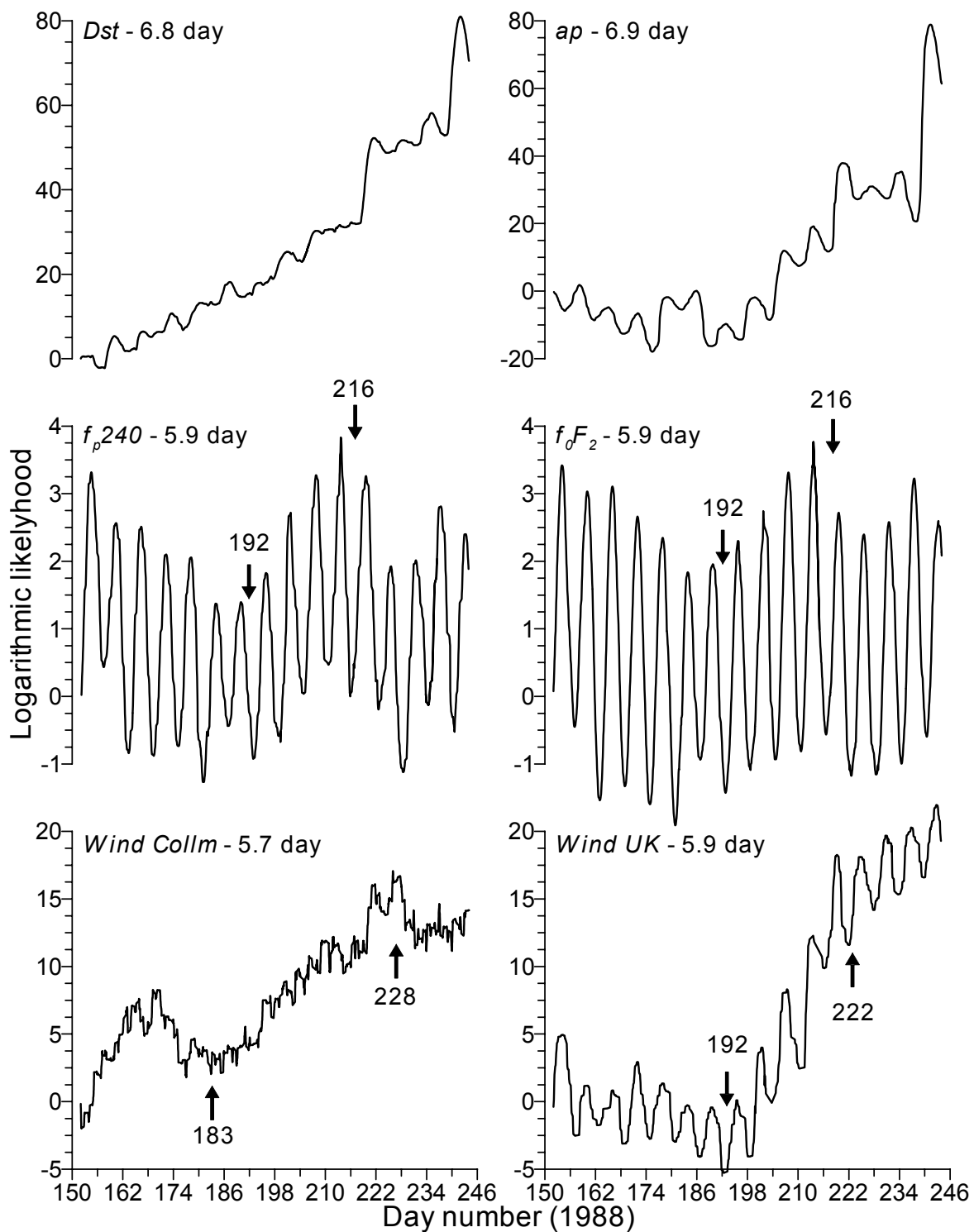

Fig. 5. Time development of the logarithmic likelihood for the dominant periods of each time series in the time interval from 1 June to 31 August 1998. The arrows in the plots indicate the time interval when the oscillations are in operation.

the plasma frequency at $240 \mathrm{~km}$. Looking at the significant periods we observe coinciding oscillation activity in the MLT and ionosphere at periods of 6 days. The dominant oscillation activity in the geomagnetic activity is at periods of 7 days. Therefore, it is reasonable to think of the oscillation activity in the MLT as a likely cause of the same oscillation activity in the ionosphere, the 7-day oscillation in the geomagnetic activity being a less likely cause for the 6-day oscillation in the ionosphere. To assess the time interval when the oscillation activity is really in operation within the time interval under study, we obtain the time development of the logarithmic likelihood for the dominant periods of the aforementioned time series (Fig. 5). Looking in detail at Fig. 5 we observe the following interesting results. The 5.7-day oscillation above Collm exists from day number 152 to 168 , then it disappears until day 183, and it becomes active again from day 183 to 228 . As already mentioned the logarithmic likelihood increases from day number 192 to 222 for the 5.9-day oscillation in the meridional wind above UK. We consider the oscillation activity with a period of about 6 days in the MLT winds to be in operation during the time interval from day number 192 to 222, when it coexists above the two wind stations. Looking at the results of the ionospheric parameters, we find that a 5.9-day oscillation becomes active from day 192 to 216 , then the logarithmic likelihood decreases sharply and the 5.9 oscillation disappears in the ionospheric parameters. The results of the geomagnetic activity indexes display a quite different pattern. Whereas a 6.8-day oscillation seems to be continuously in operation, with the Dst index being enhanced at day 218 and 240, the logarithmic likelihood for the 6.9-day oscillation in the Ap index increases from day 197 to 222 , with two sharp enhancements at day 218 and 

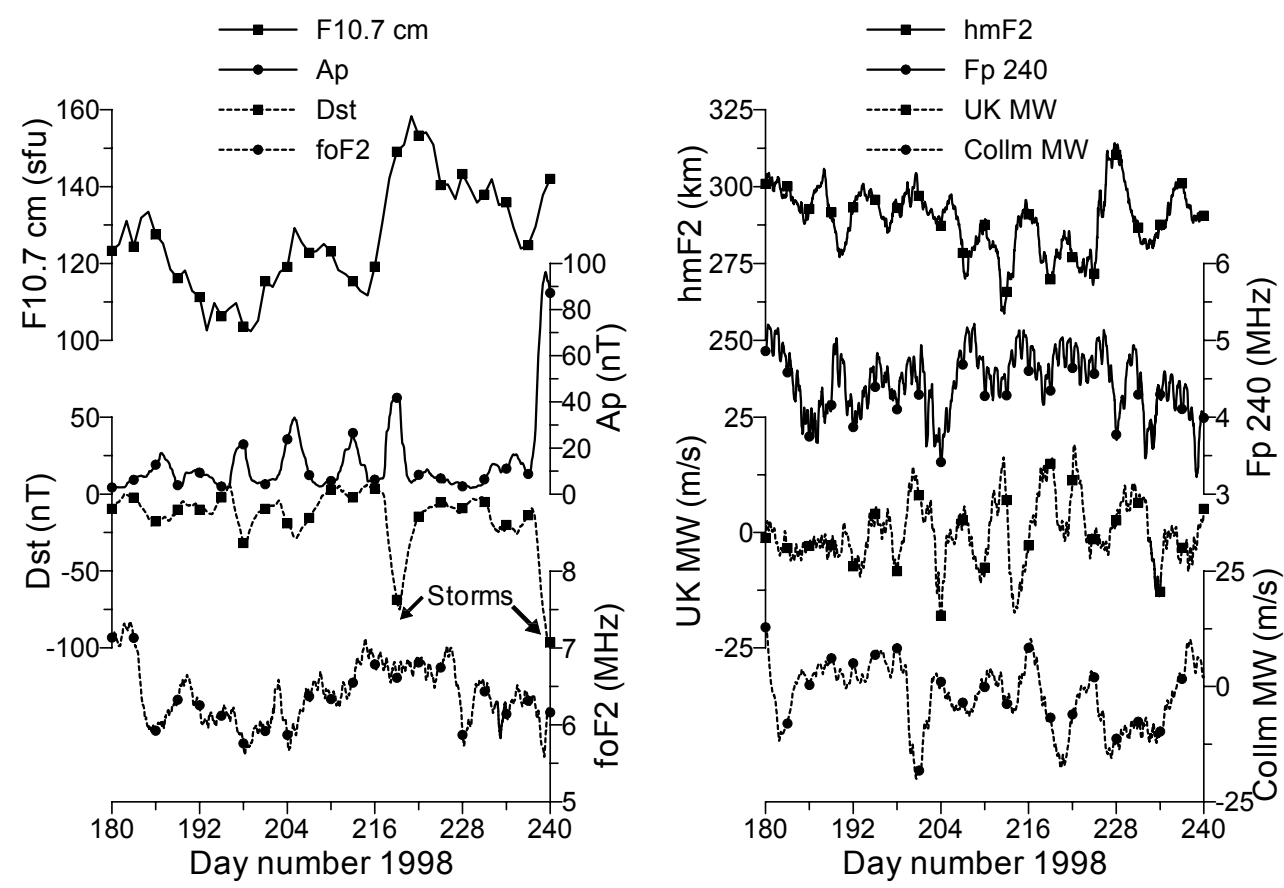

Fig. 6. Time development of the 51-h running mean values of each time series in the time interval from 1 June to 31 August 1998.

240. So, we may conclude that a 6-day oscillation becomes active in the ionosphere when a similar oscillation activity is in operation in the MLT winds. However, the oscillation in the ionosphere has a shorter life time; it finished at day 216, approximately one wave period earlier than in the MLT winds. We will try to explain later a plausible cause of the shorter lifetime of that oscillation activity in the ionosphere compared with MLT winds.

The results discussed above can be confirmed by observations from the time development of the parameters under study showed in Fig. 6, where the smoothed data with a 51-h running mean are depicted. Here we observe clearly the oscillation with a period near 6 days in the meridional winds from day 192 to 222 above UK and from day 183 to 228 above Collm. We also observe a 6-day oscillation in the ionospheric parameters from day 192 to 216 approximately, that is better expressed in the $f_{p} 240$. In addition to that, we observe a clear enhancement of the geomagnetic activity at days 217-218 and at days 239-240 $\left(A_{p}\right)$, which is related with large geomagnetic storms $\left(D_{s t}\right)$. These geomagnetic disturbances coincide in time with the sharp enhancements of the logarithmic likelihood observed in the geomagnetic activity indexes (Fig. 5). In addition to that, the 6-day oscillation activity of the ionospheric parameters just ends at the time of the first geomagnetic storm (Fig. 5). Thus, we think that the ionospheric effects of the first geomagnetic storm mask the assumed effects in the ionosphere of the oscillation activity in the MLT wind, being a candidate for explaining why the 6-day oscillation in the ionosphere has a lifetime shorter than in the MLT.

\section{Westward propagating 6-day wave of the electron density in the F2-peak}

To assess the existence of wave structures in the ionosphere, we evaluate the oscillation activity of $f o \mathrm{~F} 2$ recorded at several ionospheric stations longitudinally distributed in the Northern Hemisphere (Table 1). We apply the Lomb-Scargle periodogram as described by Hocke (1998) to the 23 time series of $f_{o} \mathrm{~F} 2$ in the periodic range from 3 to 8 days for the time interval between day number 190 and 225, in order to obtain their amplitude and phase spectra. Figure 7 depicts the amplitude spectra as a function of period and longitude. We choose that time interval because then oscillation activity with a period near 6 days in the MLT and ionosphere exists. We look for the oscillation activity near a period of about 6 days that exists at global or large scale and behaves as a wave. To assure that and as a first standard of judgement, the dominant period of oscillation at individual stations should not spread more than 10 hours from the median value of the dominant period of the time series. We find that the median value of the dominant period is 6.1 days and dashed lines in Fig. 7 show the allowed limits for the dominant oscillating period. Only 5 time series of the 23 under study did not match our first standard. This fact could be the result of the poor quality data for these stations that have many gaps. These time series are those marked with an asterisk in Fig. 7 and they will be not considered for further analyses. As a second standard the phase of oscillation as a function of the longitude should be a smooth function. Then we assume the oscillation activity of the $f o \mathrm{~F} 2$ as a cosine function with a period of about 6 days, 


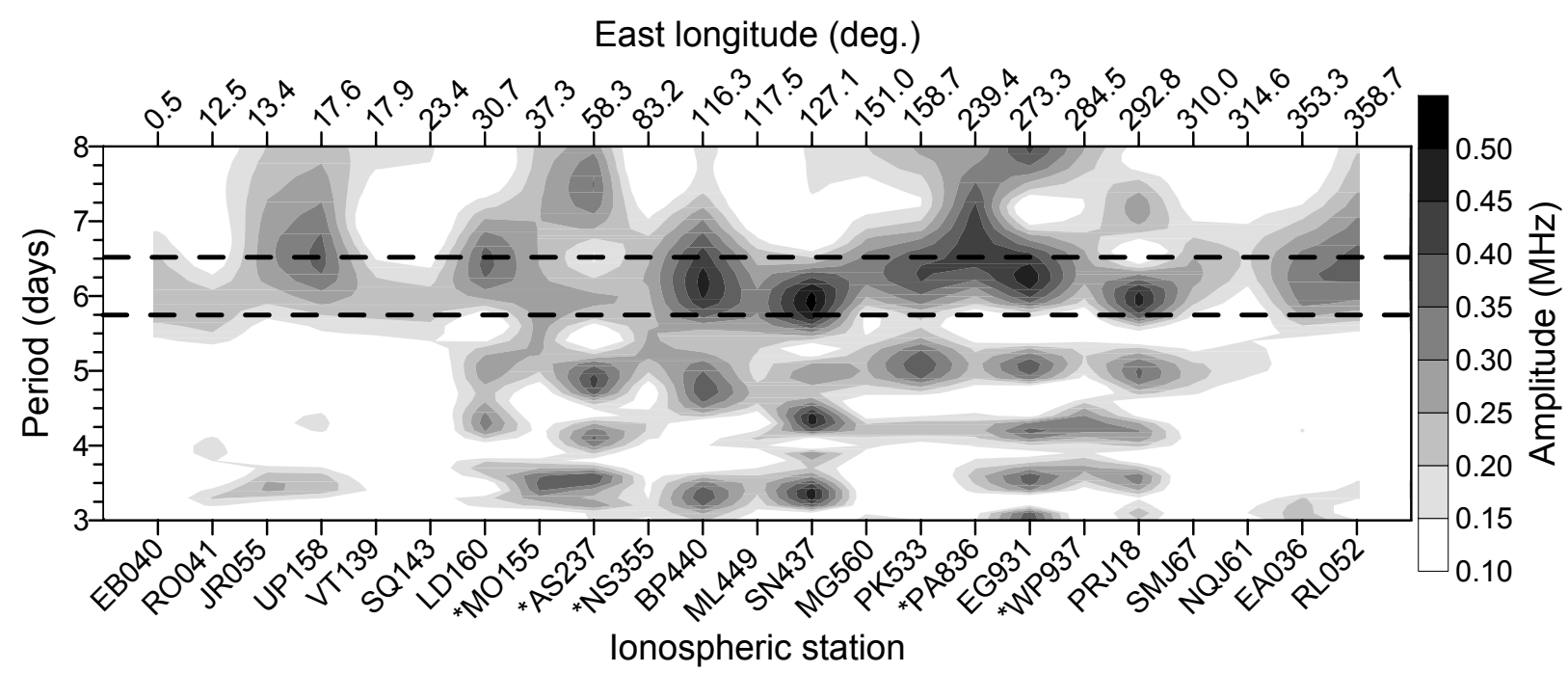

Fig. 7. Period-longitude cross sections of the amplitude spectra of the $f o F 2$, note that the longitude $x$-axis is not equally spaced. Amplitudes above $0.2 \mathrm{MHz}$ are statistically significant above the 0.95 confidence level. See text for details.

$f_{o} \mathrm{~F} 2(t, \varphi)=A \times \cos (\omega t-\varphi(\lambda))$.

From Eq. (2) we may obtain the zonal wave number, $s=$ $\partial \varphi / \partial \lambda$, where $\varphi$ is the phase of the oscillation in the ionospheric station at a longitude $\lambda$. Positive values of $s$ indicate a westward traveling wave, negative values indicate eastward, and zero values of $s$ indicate standing waves. We obtain $s$ by fitting the pairs $(\varphi, \lambda)$ to a straight line and by assuming the slope of the linear fitting equals $s$. Moreover, the obtained values of s must be very close to integer, in order to have physically consistent results. However, there are two problems when we look for the best linear fit of the phases as a function of longitude. One problem is due to the uncertainty of $2 \pi$ that arises when a new cycle of oscillation starts within a longitude sector. The other problem is due to the longitude distribution of the ionospheric stations that arises from the quality and availability of data, and from large gaps due to the oceans. Due to the latter, the data available are clustered in three sectors, West Europe $\left(-10^{\circ} \mathrm{E}-30^{\circ} \mathrm{E}\right)$, East Asia $\left(115^{\circ} \mathrm{E}-160^{\circ} \mathrm{E}\right)$, and North America $\left(270^{\circ} \mathrm{E}-315^{\circ} \mathrm{E}\right)$. We avoid these problems by constructing two data sets of pairs $(\varphi, \lambda)$, in one data set we added one cycle to the phases. Then we combine these two data sets and from them we build a working data set that assures the second standard of judgement. After that we find the best linear fit (top plot of Fig. 8), where the open circles have not been considered in the fit. The main reason why we disregarded the pairs $(\varphi, \lambda)$ corresponding to the ionospheric stations JR055 and ML449 from the linear fit is because these pairs become separated from the main trend in their respective longitude sectors. The pairs $(\varphi, \lambda)$ corresponding to the ionospheric stations EG931 and PRJ18 have been not considered to avoid the problem of clustering in the fit. The slope of the best fit, as depicted in the top plot of Fig. 8 is 1.83 , very close to 2 . Then it is likely that we observe a 6-day wave-like oscillation westward, propagating with $s=2$ at the maximum electron density of the ionospheric F-region. In addition to that, if we suppose the origin of the phase of the 6-day wave that obtained for the ionospheric station RL052 and we build the function $\varphi(\lambda)$ ), assuming a westward propagating wave with $s=2$, the agreement between the aforementioned phase function and the obtained pairs $(\varphi, \lambda)$ is excellent (bottom plot of Fig. 8). So, we may conclude that we find a 6-day westward propagating wave-like oscillation with $s=2$ in the ionosphere that is simultaneously in operation with 6-day oscillation activity in the MLT. Because no similar oscillation is present in the geomagnetic activity variations, indeed it is not of geomagnetic origin. So, it is possible that this event is forced from the wave activity in the MLT neutral winds.

\section{Linking mechanisms between the wave activity in the MLT and ionosphere}

It is accepted that $\mathrm{PW}$ propagate from the lower atmosphere and they may reach the MLT, but model evaluations do not predict significant penetration of these waves above 100 $110 \mathrm{~km}$ (Hagan et al., 1993). Meyer and Forbes (1997b) propose the suppression of the spectral response to the thermospheric forcing for the eastward/westward propagating waves with periods larger than $24 / 48 \mathrm{~h}$ by ion drag as a cause for that. However, PW type oscillations are frequently observed in the thermosphere and ionosphere (Canziani, 1994; Pancheva et al., 1994). Also, vertical propagating wave events are reported in the literature at low-latitude E-region and at middle-latitude F-region (Zhou et al., 1997; Altadill and Apostolov, 1998). Although the above results are mainly for quasi-2-day oscillations, we showed the existence of upward propagating wave events with periods of about 6 days in the ionospheric F-region that were coherent with the wave 

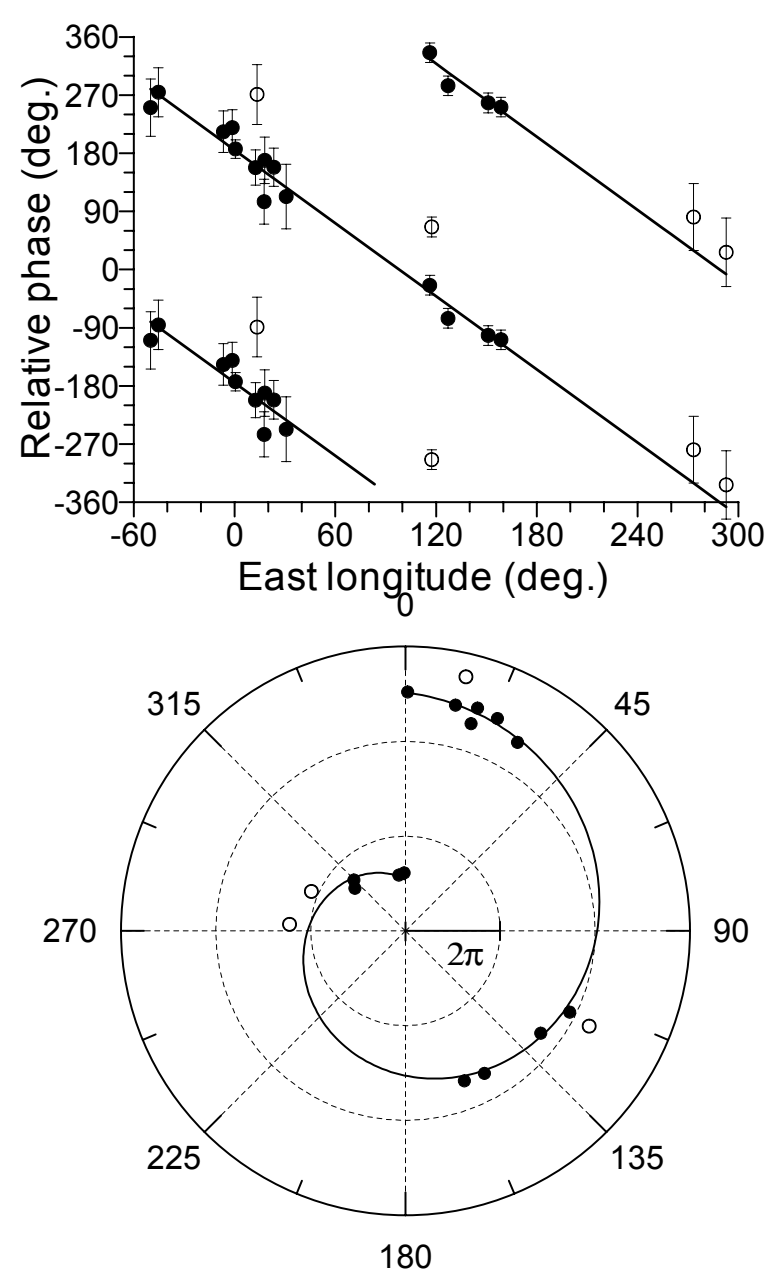

Fig. 8. The plot at the top depicts the phases of the 6-day oscillation in the $f o \mathrm{~F} 2$ as a function of the longitude (open and full circles). The straight lines in the top plot mean the best linear fit. The polar plot at the bottom depicts the phase (radius) as a function of the longitude (angle), where the line means the assumed phase function $\varphi(\lambda)$ for a westward propagating wave with $s=2$ and the dots (open circles) represent the measured phases by taking into account that a new cycle of oscillation starts within a longitude sector. See text for details.

activity in the MLT (Altadill and Apostolov, 2001; Altadill et al., 2001b). Due to that several investigations propose mechanisms of indirect forcing of the PW activity in the MLT on the ionosphere.

Recent papers propose the PW interaction or modulation of upward propagating tides and gravity waves to explain the penetration of the PW activity into the thermosphere/ionosphere system (Mitchell et al., 1996; Meyer, 1999). Also, the interaction of the PW at dynamo altitudes with the strong diurnal/semidiurnal dependence of the ionosphere may be a cause of the observed wave activity in the ionosphere (Forbes and Zhang, 1997; Pancheva et al., 2002). We evaluated the possible existence of PW interaction or modulation of tides in the MLT winds and in the ionospheric data. We have found that such interaction or modulation does

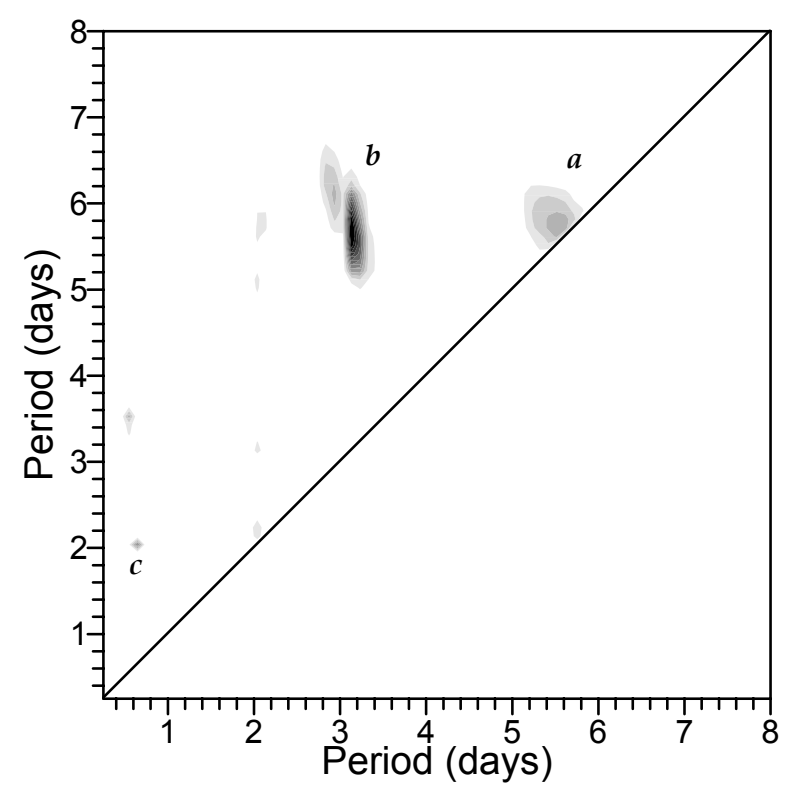

Fig. 9. Magnitude of the bispectrum squared of the UK meridional wind data. Only the top left half of the quadrant is plotted because the bispectrum is symmetrical about a diagonal.

not exist in this case. In order to assess that we applied bispectral analysis to the wind and ionospheric data, and we evaluated the time development of the 12- and 24-h oscillations in both wind and ionospheric data.

Figure 9 depicts the bispectrum of the UK meridional wind data in the periodic domain. The bispectrum was obtained from 14 intervals of 40-day duration shifted by 2 days from one to the other. The bispectrum estimator shows significant signals around interaction periods between 5.8 and 5.8 days (Fig. 9a), between 2.9 and 5.8 days (Fig. 9b), and between 2 and 0.5 days (Fig. 9c), others are practically below the noise level. We think that the interaction signals $a$ and $b$ are equivalent, indicating the interaction sum triplet 5.8/5.8/2.9 and the difference triplet 2.9/5.8/5.8. Then it reasonable to think that the 2.9 day period involved in the aforementioned interaction triplets represents the first harmonic of the 5.8 period (Kim and Powers, 1979). Another possibility for signal b could be the sum triplet 2.9/5.8/1.9, but this would mean that the 2-day period in the MLT is the result of interaction between 3 - and 6-day periods, which is very unlikely. Finally, signal $c$ indicates the possibility of interaction sum triplet 2/0.5/0.4 and difference triplet $2 / 0.5 / 0.67$. Although not shown here, we find an equivalent result for the Collm meridional wind data, where only signal $\mathrm{c}$ was significant in bispectrum estimator. So, we do not find significant interaction between periods of about 6 days and periods of diurnal/semidiurnal tide in the MLT. These results are in agreement with previous results found in the MLT (Jacobi et al., 2001). We also applied bispectral analysis to the ionospheric data, as already mentioned, and we did not find a signal of interaction between periods of about 6 days and periods of diurnal/semidiurnal dependence of the ionosphere in the bispectrum. 
Figure 10 displays the time development of the amplitudes corresponding to the 12- and 24-h oscillations for both the meridional wind component in the UK and the plasma frequency at $240 \mathrm{~km}$ in the Observatorio del Ebro. The shaded region of Fig. 10 enhances the time interval when the 6-day event was found to be in operation. The amplitudes were computed from time intervals with duration 6 times the period under study and shifted by one hour from one to the other. We used for that the Lomb-Scargle periodogram as described by Hocke (1998). From Fig. 10 we do not observe any amplitude modulation by a period of 6 days in wind or in ionospheric data. We can observe only a $\sim 10$ day modulation of the amplitude of the 24-h oscillation in winds (Fig. 10c) and may also be of the amplitude of the 24h. oscillation in the plasma frequency (Fig. 10d).

As a conclusion of the above results, neither the PW interaction or modulation of tides in the MLT nor the interaction of waves at dynamo altitudes with the strong diurnal/semidiurnal dependence of the ionosphere seem to be likely candidates to explain the 6-day wave-like oscillation we observe in the ionosphere. However, we cannot evaluate the role of the "gravity wave" mechanism proposed by Meyer (1999) due to the data we use. So, it is an open question whether such mechanism may play a role for transmitting PW activity from the MLT to the ionosphere in this case.

Another plausible mechanism to explain the PW type oscillations in the ionospheric F-region involves the ionospheric effects of the geomagnetic storms. It is accepted that strong geomagnetic storms provide significant changes in the thermospheric composition at middle latitudes, especially the ratio of atomic versus molecular species (FullerRowell et al., 1996). These changes generate negative ionospheric storms, and they may provide dramatic changes in the electron density of the ionospheric F-region, mainly in summer thermosphere. Therefore, a sequence of geomagnetic storms and substorms that repeats at periods of PW could reflect PW type oscillations in the ionosphere (Altadill et al., 2001b). However, we may discard this mechanism for the 6day wave-like oscillation we find in the ionosphere, because simultaneous oscillation activity in the geomagnetic activity at periods of about 6 days does not exist. Also, strong geomagnetic storms may generate pseudo-diurnal harmonics or in situ tides in the ionosphere (Fuller-Rowell et al., 2000). Their interaction can then account for wave-like oscillations in the ionosphere (Altadill et al., 2001b; Pancheva et al., 2002). We do not think that the above mechanism is a likely candidate to explain the 6-day wave-like oscillation we observe in the ionosphere because we do not find a signal of interaction between periods of about 6 days and periods of pseudo-diurnal dependence of ionospheric parameters in the bispectrum.

We think that the wave activity at periods of about 6 days in the MLT winds can be the source of the 6-day wave-like oscillation we observe in the ionosphere. Pancheva and Lysenko (1988) discussed two possible mechanisms that can explain the influence of PW in the MLT on the ionosphere. One of them was proposed to explain the quasi-2-day oscilla-

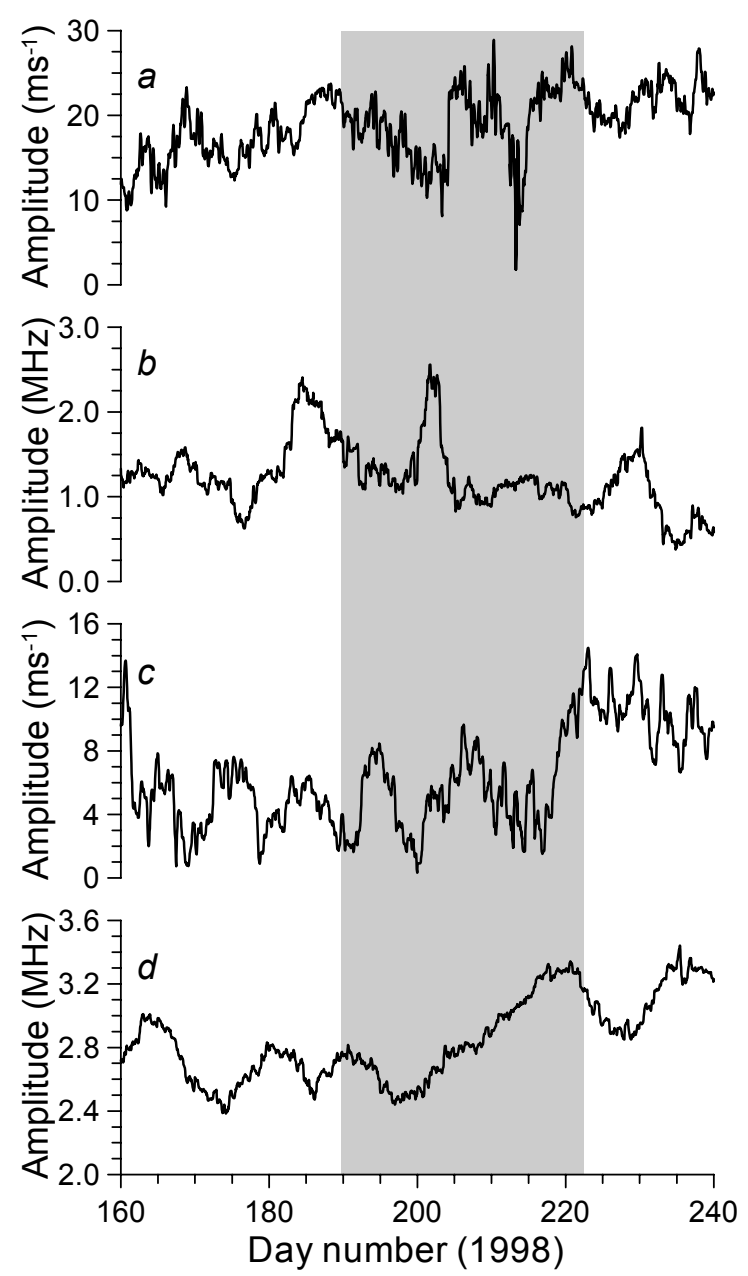

Fig. 10. Time development of the amplitudes of the 12-h oscillation of the meridional wind (a) and of the plasma frequency at $240 \mathrm{~km}$ (b), and of the amplitudes of the 24-h oscillation of the meridional wind (c) and of the plasma frequency at $240 \mathrm{~km}$ (d).

tions (Mikhailov, 1983) and it proposes that PW in the MLT wind may cause a redistribution of $[\mathrm{O}],\left[\mathrm{O}_{2}\right]$ and $\left[\mathrm{N}_{2}\right]$ at the base of the thermosphere and to change the mean vertical velocity of mass transport. This velocity was found to be $u \sim-2 \mathrm{~cm} / \mathrm{s}$ and it affects the vertical transport of minor constituents and provides in phase changes of the electron density at the F2- and E-peaks. The other mechanism discussed by Pancheva and Lysenko (1988) assumes that the PW in the MLT may induce electric fields via ionospheric wind dynamo (Ito et al., 1986). These electric fields could generate then the same oscillation activity in the "vertical plasma drift" and in the plasma density of the ionospheric F-region. However, model results suggest that the wind magnitudes have to be on the order of a few tens of $\mathrm{m} / \mathrm{s}$ in order to validate that mechanism (Chen, 1992). The amplitudes of the 6-day oscillations we observe in the MLT winds as obtained from the amplitude spectra (Hocke, 1998) are $10 \mathrm{~m} / \mathrm{s}$ for UK data and $5 \mathrm{~m} / \mathrm{s}$ for Collm data. It seems that they are not strong enough, but we must take into account that these amplitudes 
are obtained by fitting the recorded wind data to a pure sinusoid function and the obtained amplitudes are the mean amplitudes for the time interval under analysis. Otherwise, we observe in the smoothed wind data favorable oscillations from -20 to $20 \mathrm{~m} / \mathrm{s}$ in UK (Fig. 3) and from -18 to $15 \mathrm{~m} / \mathrm{s}$ in Collm (Fig. 6). Then, according to that, the oscillation activity at periods of about 6 days in the meridional wind would probably generate electrodynamic effects and would be the source of the 6-day wavelike oscillation we observe in the ionosphere. Although we did not find oscillation activity at periods of about 6 days in the zonal wind, Lastovicka and Sauli (1999) have found the meridional wind component to be better for relating the F2-region than the zonal wind as to PW type oscillations. In addition to these results and based on analyses of the electron density profiles above a single ionospheric station (Altadill et al., 2001b), we showed that the 6-day wave event studied here displays a clear propagation from below. We also showed a coherency between the time series related to the chain MLT-Dynamo-Ionosphere.

\section{Summary and conclusions}

Mid-latitude ionospheric measurements at the Northern Hemisphere during summer 1998 from a variety of ionosondes have been investigated with respect to a traveling 6-day wave-like oscillation in the ionosphere. From the phase comparisons of the $f o \mathrm{~F} 2$ this oscillation has been identified as a westward propagating wave number 2 . Simultaneous neutral wind measurements reveal a 6-day oscillation in the meridional winds at the MLT, indication of a connection between the ionosphere and the neutral atmosphere.

We evaluated the potential mechanisms that can account for PW type oscillations in the ionosphere. As a conclusion, taking into account previous results and the results obtained here, the global scale 6-day westward propagating wavelike oscillation in the maximum electron density of the ionosphere is obviously forced by the wave activity in the MLT winds at periods of about 6 days. As discussed in Sect. 6, we think that the "vertical plasma drift" is the most likely candidate for transmitting the PW activity from the MLT up to the ionospheric F2-region in this case. However, it remains an open question whether the "gravity wave" mechanism may also play a role.

Both mechanisms "vertical plasma drift" and "gravity wave" would explain the forcing of the 6-day wave-like oscillation in the $f o \mathrm{~F} 2$ from the wave activity in the MLT, but there is a disclosure between the zonal structure in the MLT and in the ionosphere. We have found that the 6-day oscillation in the MLT winds may be a westward propagating wave with zonal wave number 4 . As already mentioned, the latest result may be spurious because we have only two wind stations that are very close to each other and because of the different type of measurements. So, we may only speculate about the zonal structure of this event in the MLT. If we assume it is true, we may propose the highest phase velocity in the ionosphere to be associated with the faster thermospheric circulation, as compared with that in the mesosphere, but it is still unclear how this would transform the wave number 4 in the MLT to wave number two in the F-region. However, wave structures with period of 6-7 days have been found in the wind regime of the MLT (Kamalabadi et al., 1997; Riggin et al., 1997), with a westward propagating wave number 1 , and it has been concluded that these waves are global scale unstable modes (Meyer and Forbes 1997a; Clark et al., 2002). Then, the oscillation activity of a period about 6 days that we observed in the MLT could likely be the effect of the aforementioned wave structures and the above discussion has not sense.

There is a plausible explanation as to why the 6-day wave event we found in the $f o \mathrm{~F} 2$ has a different zonal structure from the wind oscillation. The arising of the 6-day wave event in the ionospheric F2-region may be induced by the forcing of such an oscillation in the MLT in a particular region of the globe, with further independent development in the F2-region. A similar mechanism has been proposed in order to explain the different zonal structure of the quasi2-day oscillation between the MLT and the ionospheric Fregion (Apostolov et al., 1995). However, there was a certain premise for such a possibility regarding the 2-day wave in the MLT (Poole, 1990) that has not been evaluated for the 67-day wave structures. Therefore, further research efforts are needed to solve the nature of these mechanisms, as well as to deepen the knowledge of the ionosphere-atmosphere interactions.

Acknowledgements. This work has been carried out under the project BTE2000-0825 (MCYT, Spain). The authors thank Dierk Kürschner, Collm, and Heinz G. Muller, Castle Eaton, for providing the wind measurements.

Topical Editor M. Lester thanks J. Laštovička and another referee for their help in evaluating this paper.

\section{References}

Altadill, D.: Planetary wave type oscillations in the ionospheric Fregion, Adv. Space Res., 26 (8), 1287-1296, 2000.

Altadill D. and Apostolov, E. M.: Vertical development of the 2-day wave in the middle latitude ionospheric F-region, J. Geophys. Res., 103, 29 199-29 106, 1998.

Altadill D. and Apostolov, E. M.: Vertical propagating signatures of wave type oscillations (2- and 6.5-days) in the ionosphere obtained from electron density profiles, J. Atmos. Sol.-Terr. Phys., 63 (9), 823-834, 2001.

Altadill, D., Apostolov, E. M., and Alberca, L. F.: Some seasonal hemispheric similarities in $f_{o} \mathrm{~F} 2$ quasi-2-day oscillations, J. Geophys. Res., 102, 9737-9739, 1997.

Altadill, D., Apostolov, E. M., Solé, J. G., and Alberca, L. F.: Preliminary results of the zonal structure of 6 days oscillation in the foF2 at middle latitude Northern Hemisphere, Bulg. Geophys. J., 27 (1-4), 115-123, 2001a.

Altadill, D., Apostolov,E. M., Solé, J. G., and Jacobi, Ch.: Origin and development of vertical propagating oscillations with periods of planetary waves in the ionospheric F-region, Phys. Chem. Earth (C), 26(6), 387-393, 2001b. 
Apostolov, E. M. and Altadill, D.: Ten periodic bands of $f o \mathrm{~F} 2$ quasiperiodic oscillations from 2 to 35 days, Bulg. Geophys. J., 21 (3), 20-24, 1996.

Apostolov, E. M., Altadill, D., and Alberca, L. F.: Characteristics of quasi-2-day oscillations in the foF2 at northern middle latitudes, J. Geophys. Res., 100, 12 163-12 171, 1995.

Apostolov, E. M., Altadill, D., and Hanbaba, R.: Spectral energy contributions of quasi-periodic oscillations (2-35 days) to the variability of the foF2, Ann. Geophysicae, 16, 168-175, 1998.

Bai, T.: Methods of periodicity analysis: Relationship between the Rayleigh analysis and a maximum likelihood method, Astrophys. J., 397, 584-590, 1992.

Bai, T. and Cliver, E. W.: A 154 day periodicity in the occurrence rate of proton flares, Astrophys. J., 363, 299-309, 1990.

Canziani, P. O.: On tidal variability and existence of planetary wave-like oscillations in the upper thermosphere - II. Non linear interactions and global scale oscillations, J. Atmos. Terr. Phys., 66, 913-930, 1994.

Chen, P. R.: Two-day oscillation of the equatorial ionisation anomaly, J. Geophys. Res., 97, 6343-6357, 1992

Clark, R. R., Burrage, M. D., Franke, S. J., Manson, A. H., Meek, C. E., Mitchell, N. J., and Muller, H. G.: Observations of 7-d planetary waves with MLT radars and UARS-HRDI instrument, J. Atmos. Sol.-Terr. Phys., 64, 1217-1228, 2002.

Forbes, J. M.: Planetary waves in the thermosphere-ionosphere system, J. Geomag. Geoelec., 48, 91-98, 1996.

Forbes, J. M. and Zhang, X.: Quasi 2-day oscillation of the ionosphere: A statistical study, J. Atmos. Sol.-Terr. Phys., 59, 10251034, 1997.

Forbes, J. M., Guffee, R., Zhang, X., Fritts, D., Riggin, D., Manson, A., Meek, C., and Vincent, R. A.: Quasi-2-day oscillation of the ionosphere during summer 1992, J. Geophys. Res., 102, 73017305, 1997.

Forbes, J. M., Palo, S. E., and Zhang, X.: Variability of the ionosphere, J. Atmos. Sol.-Terr. Phys., 62, 685-693, 2000.

Fuller-Rowell, T. J., Codrescu, M. V., Risbeth, H., Moffet, R. J., and Quegan, S.: On the seasonal response of the thermosphere and ionosphere to geomagnetic storms, J. Geophys. Res., 101, 2343-2353, 1996.

Fuller-Rowell, T. J., Codrescu, M. V., and Wilkinson, M.: Quantitative modelling of the ionospheric response to geomagnetic activity, Ann. Geophysicae, 18, 766-781, 2000.

Hagan, M. E., Forbes, J. M., and Vial, F.: Numerical investigations of the propagation of the quasi-two-day wave into the lower thermosphere, J. Geophys. Res., 98, 23 193-23 205, 1993.

Harris, T. J.: A long-term study of the quasi-two-day wave in the middle atmosphere, J. Atmos. Terr. Phys., 56 (5), 569-579, 1994.

Hocke, K.: Phase estimation with the Lomb-Scargle periodogram method, Ann. Geophysicae, 16, 356-358, 1998.

Horne, J. H. and Baliunas, S. L.: A prescription for period analysis of unevenly sampled time series, Astrophys. J., 302, 757-763, 1986.

Huang, X. and Reinisch, B. W.: Vertical electron density profiles from the digisonde network, Adv. Space Res., 18 (6), 121-129, 1996.

Ito, R., Kato, S., and Tsuda, T.: Consideration of an ionospheric wind dynamo driven by a planetary wave with a two-day period, J. Atmos. Terr. Phys., 48, 1-13, 1986.

Jacobi, Ch., Portnyagin, Yu. I., Merzlyakov, E. G., Kashcheyev, B. L., Oleynikov, A. N., Kürschner, D., Mitchell, N. J., Middleton, H. R., Muller, H. G., and Comley, V. E.: Mesosphere/lower thermosphere wind measurements over Europe in summer 1998,
J. Atmos. Sol.-Terr. Phys., 63, 1017-1031, 2001.

Kamalabadi, F., Forbes, J. M., Makarov, N. M., and Portnyagin, Yu. I.: Evidence for nonlinear coupling of planetary waves and tides in the antarctic mesopause, J. Geophys. Res., 102 (D4), 4437-4446, 1997.

Kim, Y. C. and Powers, E. J.: Digital bispectral analysis and its applications to nonlinear wave interactions, IEEE Transactions on Plasma Sciences PS-7 (2), 120-131, 1979.

Lastovicka, J. and Sauli, P.: Are the PW type oscillations in the F2-region caused by planetary wave modulation of upward propagating tides?, Adv. Space Res., 24, 1473-1476, 1999.

Meyer, C. K.: Gravity waves interaction with planetary waves: A mechanism for penetration into the thermosphere-ionosphere system, J. Geophys. Res., 104, 28 181-28 196, 1999.

Meyer, C. K. and Forbes, J. M.: A 6.5-day westward propagating planetary wave: Origin and characteristics, J. Geophys. Res., 102 (D22), 26 173-26 178, 1997a.

Meyer, C. K. and Forbes, J. M.: Natural oscillations of the ionosphere-thermosphere-mesosphere (ITM) system, J. Atmos. Sol.-Terr. Phys., 59, 2185-2202, 1997 b.

Mikhailov, A. V.: Mechanism for in-phase variations of electron concentration between E- and F2-layers (in Russian), Geomagn. Aeronomy, 23, 557-561, 1983.

Mitchell, N. J., Williams, P. S. J., Beard, A. G., Buesnell, G. R., and Muller, H. G.: Nonlinear planetary/tidal wave interactions in the lower thermosphere observed by meteor radar, Ann. Geophysicae, 14, 364-366, 1996.

Muller, H. G., Havill, R. L., Comley, V. E., and Hill, P. C. J.: A study of meteor radar winds from two locations in the British Isles, J. Atmos. Terr. Phys., 57, 979-993, 1995.

Pancheva, D. and Lastovicka, J.: Planetary wave activity in the lower ionosphere during CRISTA I campaign in autumn (October-November). Ann. Geophysicae, 16, 1014-1023, 1998.

Pancheva, D. and Lysenko, I.: Quasi-two-day fluctuations observed in the summer F-region electron maximum, Bulg. Geophys. J., 14 (2), 41-51, 1988.

Pancheva, D., Alberca, L. F., and de la Morena, B. A.: Simultaneous observations of quasi-two-day variations in the lower and upper ionosphere over Europe, J. Atmos. Terr. Phys., 56, 43-50, 1994.

Pancheva, D., Mitchell, N., Clark, R. R., Drobjeva, J., and Lastovicka, J.: Variability in the maximum height of the ionospheric F2-layer over Millstone Hill (September 1998 - March 2000); influence from below and above, Ann. Geophysicae, 20, 1807 1819, 2002.

Poole, L. M. G.: The characteristics of the mesospheric two-day wave as observed at Grahamstown $\left(33.3^{\circ} \mathrm{S}, 26.5^{\circ} \mathrm{E}\right)$, J. Atmos Terr. Phys., 52, 259-268, 1990.

Riggin, D. M., Fritts, D. C., Tsuda, T., Nakamura, T., and Vincent, R. A.: Radar observations of a 3-day Kelvin wave in the equatorial mesosphere, J. Geophys. Res., 102 (D22), 26 141-26 175, 1997.

Rishbeth, H. and Mendillo, M.: Patterns of F2-layer variability, J. Atmos. Sol.-Terr. Phys., 63, 1661-1680, 2001.

Salby, M. L.: Survey of planetary-scale travelling waves: The state of theory and observation, Rev. Geophys., 22, 209-236, 1984.

Scargle, J. D.: Studies in astronomical time series analysis. II. Statistical aspects of spectral analysis of unevenly spaced data, Astrophys. J., 263, 835-853, 1982.

Schminder, R. and Kürschner, D.: Permanent monitoring of the upper mesosphere and lower thermosphere wind fields (prevailing and semidiurnal tidal components) obtained from LF D1 measurements in 1991 at the Collm Geophysical Observatory, J. At- 
mos. Terr. Phys., 56, 1263-1269, 1994.

Wu, D. L., Fishbein, E. F., Read, W. G., and Waters, J. W.: Excitation and evolution of the quasi-2-day wave observed in UARS/MLS temperature measurements, J. Atmos. Sci., 56, 728738, 1996.

Wu, D. L., Hays, P. B., and Skinner, W. R.: Observation of the 5- day wave in the mesosphere and lower thermosphere, Geophys. Res. Lett., 21 (24), 2733-2736, 1994.

Zhou, Q. H., Sulzer, M. P. and Tepley, C. A.: An analysis of tidal and planetary waves in the neutral winds and temperature observed at low latitude E-region heights, J. Geophys. Res., 102, 11 491-11 505, 1997. 